MIFP-07-15

ACT-03-07

May, 2007

\title{
No-Scale Solution to Little Hierarchy
}

\author{
Bhaskar Dutta $^{1}$, Yukihiro Mimura ${ }^{1}$ and Dimitri V. Nanopoulos ${ }^{1,2,3}$ \\ ${ }^{1}$ Department of Physics, Texas A $3 M$ University, College Station, TX 77843-4242, USA \\ ${ }^{2}$ Astroparticle Physics Group, Houston Advanced Research Center (HARC), \\ Mitchell Campus, Woodlands, TX 77381, USA \\ ${ }^{3}$ Academy of Athens, Division of Natural Sciences, \\ 28 Panepistimiou Avenue, Athens 10679, Greece
}

\begin{abstract}
We show that the little hierarchy problem can be solved in the no-scale supergravity framework. In this model the supersymmetry breaking scale is generated when the electroweak symmetry breaking condition is satisfied and therefore, unlike usual supersymmetric models, the correlation between the electroweak symmetry breaking scale and the average stop mass scale can be justified. This correlation solves the little hierarchy puzzle. Using minimal supergravity boundary conditions, we find that the parameter space predicted by no-scale supergravity is allowed by all possible experimental constraints. The predicted values of supersymmetric particle masses are low enough to be very easily accessible at the LHC. This parameter space will also be probed in the upcoming results from the dark matter direct detection experiments.
\end{abstract}




\section{Introduction}

Supersymmetry (SUSY) is one of the key ingredients to consider physics beyond the Standard Model (SM). The large scale hierarchy between the Planck scale and the weak scale is stabilized once the hierarchy is generated. In the minimal SUSY extension of the standard model (MSSM), the electroweak symmetry breaking condition is satisfied by the renormalization group running of the SUSY breaking mass for Higgs fields, and therefore, a large hierarchy can be generated radiatively [1]: $m_{W} / M_{P} \sim \exp \left(-4 \pi^{2}\right)$. In this picture we come across three different scales. The scale $Q_{0}$, where one of the eigenvalues of Higgs mass squared becomes negative, is much smaller than the Planck scale. However, to generate the electroweak symmetry breaking vacua radiatively a typical SUSY breaking scale $Q_{S}$, where loop correction from the Higgs potential vanishes, is needed to be smaller than the scale $Q_{0}$. In addition, there is another scale, $Q_{\text {st }}$, where the electroweak potential is destabilized in the $D$-flat direction. The SUSY breaking scale $Q_{S}$ needs to be within the window between $Q_{\text {st }}$ and $Q_{0}$, i.e., $Q_{\text {st }}<Q_{S}<Q_{0}$. In the SUSY breaking models, the scale $Q_{S}$ is just an input to obtain a phenomenological model as an anthropic selection.

The recent SUSY particle search limits seem to demand an unnatural constraint on the radiative electroweak symmetry breaking vacua to generate the correct $Z$ boson mass. The search attempts have already exceeded the $Z$ boson mass scale. This means that $Q_{S}$ is pushed up, and a little hierarchy between the $Z$ boson mass and SUSY breaking masses gets created. Naively, if an unnatural fine-tuning is not allowed, the electroweak symmetry breaking condition leads to the fact that $Q_{S}$, typically the average stop mass, is not very large compared to the $Z$ boson mass. Surely, if we allow fine-tuning, there is no problem. The fine-tuning is encoded in the fact that the two unrelated scales $Q_{S}$ and $Q_{0}$ are close. The scale $Q_{0}$ is obtained to be hierarchically small from the Planck scale, and the hierarchy is determined by dimensionless parameters. While the SUSY breaking scale $Q_{S}$ is a dimensionful parameter of the model. Why are two such unrelated scales destined to be close? Does there exist any relation between $Q_{0}$ and $Q_{S}$ ? These are fundamental questions and require urgent attention since the recent experimental constraints have caused a little hierarchy between the $Z$ boson and SUSY breaking masses.

It is well known that SUSY is an attractive candidate of physics beyond the SM since it can solve the unnatural tuning for the quadratic divergence of Higgs mass. It also provides a dark matter candidate, the neutralino, to explain the $23 \%$ of the content of the Universe [2]. It is therefore important to understand whether there exists a physics reason behind the selection of the electroweak symmetry breaking vacua with little hierarchy. One of the recent attempts is to reduce the fine-tuning in the symmetry breaking condition by selecting a SUSY breaking 
scenario [3, 4, 5. Another is to consider statistically probable vacua among the electroweak symmetry breaking vacua [6, 7]. Such landscape idea can nicely explain the little hierarchy. However, the selection of the symmetric breaking vacua is still due to the anthropic reason.

No-scale supergravity (SUGRA) model [8, 9], on the other hand, can explain not only the selection of the electroweak symmetry breaking window, but also the little hierarchy between the $Z$ boson mass and SUSY breaking scale [10]. In no-scale SUGRA, the gravitino mass is not determined due to the flat potential and this continues until the gravitino mass or the SUSY breaking feels the electroweak potential. The gravitino mass is determined dynamically due to the radiative electroweak symmetry breaking. In this sense, the radiative symmetry breaking vacua are automatically selected. Therefore, the reason why $Q_{S}$ is in the symmetry breaking window is explained by its own mechanism. Besides, the closeness of $Q_{0}$ and $Q_{S}$ is also realized by the feature of no-scale electroweak potential. So, the no-scale structure is a golden solution of the little hierarchy problem.

No-scale SUGRA is well studied and has been well known for more than twenty years [8, 9]. However, the no-scale structure is often used only as a boundary condition at the unification scale. In this paper, we discuss the no-scale structure of the dynamical determination of the SUSY breaking scale as a natural solution of the little hierarchy. The electroweak symmetry breaking leads to two conditions corresponding to the minimization by Higgs vacuum expectation values (VEVs). The dynamical determination of the SUSY breaking scale gives one more relation between the $Z$ boson mass and the SUSY breaking scale. The relation is written in terms of the renormalization group equations (RGEs) of the Higgs boson mass. We describe the model constraints to generate radiative electroweak symmetry breaking vacua, and find the prediction of no-scale SUGRA. Importantly, we find that the SUSY breaking mass, typically the stop and the gluino masses have upper bounds which are very easy to reach at the upcoming collider experiments. We also describe the phenomenological constraints and show the interesting prospect of discovering this model at the upcoming dark matter detection experiments.

This paper is organized as follows. In section 2, we discuss the Higgs potential and see what kind of tuning is needed. In section 3, we describe symmetry breaking vacuum and no-scale SUGRA. In section 4, we discuss no-scale supergravity model and phenomenology. Section 5 contains our conclusion. 


\section{Higgs potential and Little Hierarchy}

The tree-level neutral Higgs potential is

$$
V^{(0)}=m_{1}^{2} v_{d}^{2}+m_{2}^{2} v_{u}^{2}-\left(m_{3}^{2} v_{u} v_{d}+\text { c.c. }\right)+\frac{g_{Z}^{2}}{8}\left(v_{u}^{2}-v_{d}^{2}\right)^{2},
$$

where $v_{u}$ and $v_{d}$ are the VEVs of the neutral Higgs bosons, $H_{u}^{0}$ and $H_{d}^{0}$. The quartic coupling is obtained from $D$-term and thus the coupling is related to the gauge couplings : $g_{Z}^{2}=g^{2}+g^{\prime 2}$. The quadratic terms are given by SUSY breaking Higgs masses, $m_{H_{d}}^{2}$ and $m_{H_{u}}^{2}$, Higgsino mass $\mu$, and SUSY breaking bilinear Higgs mass $B \mu: m_{1}^{2}=m_{H_{d}}^{2}+\mu^{2}, m_{2}^{2}=m_{H_{u}}^{2}+\mu^{2}$ and $m_{3}^{2}=B \mu$. The $Z$ boson mass is $\frac{g_{Z}}{\sqrt{2}} v$, where $v=\sqrt{v_{u}^{2}+v_{d}^{2}}$. Minimizing the tree-level Higgs potential (i.e., $\left.\partial V / \partial v_{u}=0, \partial V / \partial v_{d}=0\right)$, we obtain

$$
\frac{M_{Z}^{2}}{2}=\frac{m_{1}^{2}-m_{2}^{2} \tan ^{2} \beta}{\tan ^{2} \beta-1}, \quad \sin 2 \beta=\frac{2 m_{3}^{2}}{m_{1}^{2}+m_{2}^{2}},
$$

where $\tan \beta=v_{u} / v_{d}$. The $Z$ boson mass can be also expressed as

$$
\frac{M_{Z}^{2}}{2}=-\mu^{2}+\frac{m_{H_{d}}^{2}-m_{H_{u}}^{2} \tan ^{2} \beta}{\tan ^{2} \beta-1} \equiv-\mu^{2}+M_{H}^{2} .
$$

The SUSY breaking Higgs mass $M_{H}^{2}$ is approximately $-m_{H_{u}}^{2}$ for $\tan \beta \gtrsim 10$. The electroweak symmetry can be broken by RGE flow of the Higgs mass [1]. Since the scale of $M_{H}$ is naively governed by colored SUSY particles, it is not comparable to the $Z$ boson mass using the current experimental bounds on uncolored SUSY particles, if the universal boundary condition is applied at the GUT or the Planck scale. Therefore, fine-tuning is required between $\mu^{2}$ and $M_{H}^{2}$. So, naturalness demands a model which generates smaller values of $\mu$ (corresponds to smaller $M_{H}$ ) to reduce the fine-tuning [11].

Since the mass parameters run by RGEs, it is important to note the scale where the finetuning is needed. Let us rewrite the expression of the $Z$ boson mass to see what kind of tuning is needed. The tree-level expression of $Z$ boson mass depends on scale $Q$, and thus, let us define the $Q$ dependent $m_{Z}^{2}$,

$$
m_{Z}^{2}(Q) \equiv 2 \frac{m_{1}^{2}(Q)-m_{2}^{2}(Q) \tan ^{2} \beta(Q)}{\tan ^{2} \beta(Q)-1}
$$

Taking into account the 1-loop correction of the potential [12] in $\overline{\mathrm{DR}}^{\prime}$ scheme [13],

$$
V^{(1)}=\frac{1}{64 \pi^{2}} \sum_{i}(-1)^{2 J_{i}}\left(2 J_{i}+1\right) m_{i}^{4}\left(\ln \frac{m_{i}^{2}}{Q^{2}}-\frac{3}{2}\right),
$$

where $J_{i}$ is a spin of the particle $i$ with mass $m_{i}$, we obtain

$$
M_{Z}^{2}=m_{Z}^{2}(Q)+\frac{1}{v^{2} \cos 2 \beta}\left(v_{u} \frac{\partial V^{(1)}}{\partial v_{u}}-v_{d} \frac{\partial V^{(1)}}{\partial v_{d}}\right) .
$$


This expression of $M_{Z}$ does not depend on $Q$ up to the wave function renormalization for $v_{u}$ and $v_{d}$ at one-loop order. Therefore the proper $Z$ boson mass is obtained approximately at $Q=Q_{S}$ where $\frac{\partial V^{(1)}}{\partial v_{u}}=\cot \beta \frac{\partial V^{(1)}}{\partial v_{d}}$ is satisfied, namely

$$
e Q_{S}^{2}=\prod_{i}\left(m_{i}^{2}\right)^{\frac{X_{i}}{X}}
$$

where

$$
X_{i}=\left(\frac{\partial m_{i}^{4}}{\partial v_{u}}-\cot \beta \frac{\partial m_{i}^{4}}{\partial v_{d}}\right)(-1)^{2 J_{i}}\left(2 J_{i}+1\right), \quad X=\sum_{i} X_{i}
$$

The scale $Q_{S}$ is naively the average of the stop masses. Let us define the scale $Q_{0}$ where the function $m_{Z}^{2}(Q)$ is zero, which is equivalent to the scale $m_{1}^{2} m_{2}^{2}=m_{3}^{4}$. Then the $Z$ boson mass is expressed as

$$
\left.M_{Z}^{2} \simeq \ln \frac{Q_{S}}{Q_{0}} \frac{d m_{Z}^{2}}{d \ln Q}\right|_{Q=Q_{0}}
$$

and

$$
\left.\frac{d m_{Z}^{2}}{d \ln Q}\right|_{Q=Q_{0}}=-\frac{2}{\cos ^{2} 2 \beta}\left(\frac{d m_{2}^{2}}{d \ln Q} \sin ^{2} \beta+\frac{d m_{1}^{2}}{d \ln Q} \cos ^{2} \beta-\frac{d m_{3}^{2}}{d \ln Q} \sin 2 \beta\right) .
$$

For large $\tan \beta \gtrsim 10$,

$$
M_{Z}^{2} \simeq \ln \left(\frac{Q_{0}}{Q_{S}}\right)^{2} \frac{d m_{2}^{2}}{d \ln Q}
$$

From this expression, one can find that the $Z$ boson mass is proportional to the stop mass up to a loop factor, and $Q_{0}$ and $Q_{S}$ need to be close as needed by the little hierarchy between the stop mass and $Z$ boson mass. It is important to note that the smallness of the $\mu$ parameter is not important in this expression since $\mu^{2}$ and $-m_{H_{u}}^{2}$ are canceled in RGE at $Q=Q_{0} 1$. Therefore, the little hierarchy is characterized only by the spectrum of stop masses in RGE of Higgs mass and the closeness of $Q_{0}$ and $Q_{S}$. For example, in the focus point solution [14] of minimal supergravity (mSUGRA), it may give rise to a solution of the naturalness problem if $Q_{S}$ is fixed at $\mathrm{TeV}$ scale (just below the focus point) since the $\mu$ parameter is small. However, the little hierarchy problem is not solved since the $Z$ boson mass is sensitive to $\ln Q_{0} / Q_{S}$ and the stop masses are heavy in this solution.

The radiative symmetry breaking elegantly explains the smallness of $Q_{0}$ and the focus point scale compared to the Planck scale. However, the hierarchy is determined irrespective of the overall scale parameter since RGEs are homogenous differential equations, and there is no reason that $Q_{0}$ and the focus point scales are close to $Q_{S}$ (which is proportional to the overall

\footnotetext{
${ }^{1}$ Once $Q_{S}$ is fixed, the smallness of $\mu$ (naturalness) is important for less fine-tuning in the $Z$ boson mass. However, there is no reason that $Q_{S}$ is fixed in general SUSY breaking model. If $Q_{S}$ is free, the tuning parameter is $\ln Q_{0} / Q_{S}$, and then the smallness of $\mu$ is not important for the tuning.
} 
scale). The little hierarchy problem that we are concerned about is why such unrelated scales are so close.

We can show that the closeness of $Q_{0}$ and $Q_{S}$ is probable among the electroweak symmetry breaking vacua in the landscape picture [7]. However, in such a picture, the vacua where the electroweak symmetry is not broken (namely $Q_{0}<Q_{S}$ ) are also enormously probable, and the electroweak symmetry breaking vacuum has a special existence among the multiverse. Obtaining the electroweak symmetry breaking vacua could be just for anthropic reason at this stage.

In this paper, we stress that electroweak symmetry breaking vacua with a little hierarchy is naturally obtained in no-scale SUGRA.

\section{Symmetry Breaking Vacuum and No-scale Model}

In this section, we study the origin of the electroweak symmetry breaking vacuum [8] and the natural occurrence of the closeness of $Q_{0}$ and $Q_{S}$ in no-scale electroweak potential.

In supergravity [15, 16], the SUSY breaking scale is obtained in the hidden sector physics, and thus the scales $Q_{0}$ and $Q_{S}$ are intuitively different and there is no reason that $Q_{S}$ is selected in the electroweak symmetry breaking region. In no-scale supergravity, on the other hand, the SUSY breaking scale is not determined since the potential for the moduli $T$ and their $F$-terms are completely flat. The SUSY breaking scale, which is a function of $T$, is determined by the radiative effect of the Higgs potential. Since the dynamical determination of the SUSY breaking scale is due to the electroweak radiative effect, $Q_{0}$ and $Q_{S}$ can be related in the no-scale SUGRA.

The $Q$-independent electroweak potential is given as

$$
V\left(v_{u}, v_{d}\right)=V^{(0)}\left(v_{u}, v_{d} ; Q\right)+\Delta V\left(v_{u}, v_{d} ; Q\right)
$$

where $\Delta V$ is loop correction and the Higgs VEVs-independent pieces need to be subtracted,

$$
\Delta V=V^{(1)}\left(v_{u}, v_{d} ; Q\right)-V^{(1)}(0,0 ; Q)
$$

When $Q_{S}$ is larger than $Q_{0}$, the electroweak symmetry does not break, and thus $v_{u}=v_{d}=0$ and $V=0$. If $Q_{S}$ is smaller than $Q_{0}$, the $Q$-independent potential can be negative due to the tree-level potential term. In other words, at the minimal point of the $Q$-independent potential $V\left(v_{u}, v_{d}, Q_{S}(T)\right)$ (i.e., $\partial V / \partial v_{u}=0, \partial V / \partial v_{d}=0$ and $\partial V / \partial T=0$ ), the electroweak symmetry is broken. Therefore, if there is no other hidden sector term to determine the scale $Q_{S}$, the breaking condition $Q_{0}>Q_{S}$ is automatically satisfied in this framework. Besides, as we will 


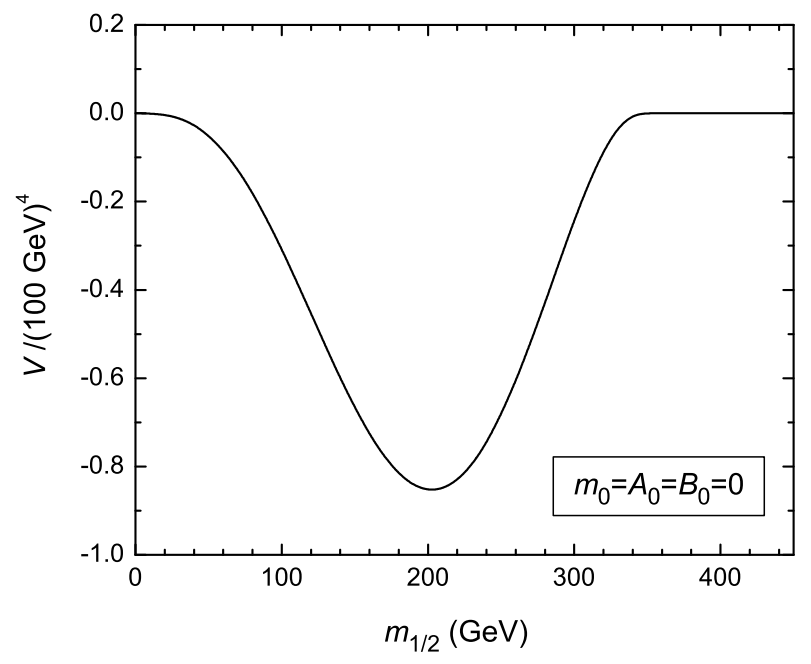

Figure 1: The no-scale electroweak potential

see later, $Q_{S}$ is just below the scale $Q_{0}$, and thus the scale $Q_{S}$ can be larger than the stabilityviolating scale $Q_{\text {st }}$.

Now let us consider a more concrete situation. We assume that every mass parameter in the supergravity model is proportional to one mass parameter (typically the gravitino mass). For example, in mSUGRA, the mass parameters are $\left(m_{0}, m_{1 / 2}, A_{0}, \mu_{0}, B_{0}\right)$, which are SUSY breaking scalar mass, gaugino mass, trilinear scalar coupling, Higgsino mass and SUSY breaking bilinear Higgs mass parameter, respectively. Since the electroweak potential does not depend on gravitino mass explicitly, it is useful to use the gaugino mass as an overall scale. A given no-scale model gives dimensionless parameters $\left(\hat{m}_{0}, \hat{A}_{0}, \hat{\mu}_{0}, \hat{B}_{0}\right)$ and $\hat{m}_{3 / 2}$ e.g., $\hat{m}_{0}=m_{0} / m_{1 / 2}$, $\hat{A}_{0}=A_{0} / m_{1 / 2}$, and so on. The overall scale $m_{1 / 2}$ is determined by the electroweak potential. In figure 1 , we show the numerically calculated potential minimized by $v_{u}$ and $v_{d}$ as a function of $m_{1 / 2}$ when $\hat{m}_{0}=\hat{A}_{0}=\hat{B}_{0}=0$. The $\hat{\mu}_{0}$ parameter is chosen to obtain the proper $Z$ boson mass at the minimum. In this choice, $\tan \beta \sim 9$ at the minimal value of the potential.

Now let us derive the fact that $Q_{S}$ and $Q_{0}$ are close at the minimal point. The potential is obtained using the minimizing conditions by $v_{u}$ and $v_{d}$ as

$$
V=-\frac{1}{2 g_{Z}^{2}} M_{Z}^{4} \cos ^{2} 2 \beta+\Delta V-\frac{1}{2}\left(v_{u} \frac{\partial \Delta V}{\partial v_{u}}+v_{d} \frac{\partial \Delta V}{\partial v_{d}}\right) .
$$

Substituting Eq.([6), we obtain

$$
V=-\frac{1}{2 g_{Z}^{2}} m_{Z}^{4}(Q) \cos ^{2} 2 \beta+\Delta V-\frac{\sin 2 \beta}{2}\left(v_{d} \frac{\partial \Delta V}{\partial v_{u}}+v_{u} \frac{\partial \Delta V}{\partial v_{d}}\right)+\cdots .
$$

Since the potential is $Q$-independent, let us choose the scale $Q$ to make terms beyond the second term to be zero. Naively, it is the scale where $\Delta V=0$ when $\tan \beta$ is large. We call this scale 
$Q_{V}$. The potential can be written as

$$
V \simeq-\frac{1}{2 g_{Z}^{2}}\left(\frac{d m_{Z}^{2}}{d \ln Q} \ln \frac{Q_{V}}{Q_{0}}\right)^{2} \cos ^{2} 2 \beta
$$

Since $\frac{d m_{Z}^{2}}{d \ln Q}$ is approximately proportional to the overall scale which is related to $Q_{V}$, the potential is

$$
V \propto-Q_{V}^{4}\left(\ln \frac{Q_{0}}{Q_{V}}\right)^{2}
$$

Minimizing the potential by $Q_{V}$, we obtain $Q_{V}=Q_{0} / e^{1 / 2}$. Thus the scale $Q_{V}$ is just below the symmetry breaking scale $Q_{0}$. When we write $Q_{S}=k Q_{V}$, the $Z$ boson mass at the minimum is obtained from Eq.(11)

$$
\left.M_{Z}^{2} \simeq\left(1-\ln k^{2}\right) \frac{d m_{2}^{2}}{d \ln Q}\right|_{Q=Q_{0}} .
$$

In the MSSM mass spectrum, the stop masses are important to determine $Q_{V}$ and $Q_{S}$. Thus, these two scales are close and $k \sim 1$. In the numerical calculation, $k$ depends on stop mixings etc, but $\ln k^{2}$ is about $0.1-0.2$. Note that the low energy particle spectrum ratio does not depend on overall scale (we choose it as $\left.m_{1 / 2}\right)$, when $\left(\hat{m}_{0}, \hat{A}_{0}, \hat{\mu}_{0}, \hat{B}_{0}\right)$ are fixed as boundary condition. Therefore, $Q_{V}$ is naively proportional to $m_{1 / 2}$, and thus, the minimization of the potential by an overall scale is rationalized.

The parameter $\hat{\mu}_{0}$ is consumed to fix $Z$ boson mass at the minimum, and $\hat{B}_{0}$ is determined when $\tan \beta$ is fixed. So, the model parameters in the minimal supergravity are $\hat{m}_{0}, \hat{A}_{0}, \tan \beta$ and the signature of $\mu_{0}$.

Since the RGE of $m_{2}^{2}$ at $Q=Q_{0}$ is almost determined by stop mass parameters with a loop factor,

$$
\frac{d m_{2}^{2}}{d \ln Q} \simeq \frac{3}{8 \pi^{2}}\left(y_{t}^{2}\left(m_{\tilde{t}_{L}}^{2}+m_{\tilde{t}_{R}}^{2}\right)+A_{t}^{2}\right)
$$

the little hierarchy between the $Z$ boson and stop masses is obtained by a minimization of the no-scale electroweak potential. Numerically one finds that the gaugino mass at the GUT scale is about $200 \mathrm{GeV}$ for small $\hat{m}_{0}$. This result does not depend on $\tan \beta$ very much unless $\tan \beta$ is small.

\section{No-scale Model and Phenomenology}

In this section, we study the no-scale supergravity model to realize the no-scale electroweak potential in the previous section, and find the phenomenological consequence of the model. There are several ways to realize the no-scale structure [8, 9]. Here, we consider the simplest model to realize what we have described in the previous section. 
In the simplest no-scale model, the Kähler potential is given as 9]

$$
\mathcal{G}=-3 \ln \left(T+\bar{T}-\frac{1}{3} \phi_{i} \bar{\phi}_{i}\right)+\ln |W|^{2},
$$

where $\phi_{i}$ 's are matter and Higgs fields. In this choice, $m_{0}$ and $A_{0}$ are zero as boundary conditions. The $\mu$ term can be proportional to gravitino mass when bilinear term $H_{u} H_{d}$ is in the Kähler potential but not in the superpotential $W$. More generally, one can write down the Kähler potential with modular weights $\lambda_{i}$ and $\rho$ as [9, 17]

$$
\mathcal{G}=-3 \ln (T+\bar{T})+\frac{\phi_{i} \bar{\phi}_{i}}{(T+\bar{T})^{\lambda_{i}}}+\frac{h}{2}\left(\frac{H_{u} H_{d}}{(T+\bar{T})^{\rho}}+\text { h.c. }\right)+\ln |W|^{2} .
$$

Then, we obtain

$$
\begin{aligned}
m_{i}^{2} & =\left(1-\lambda_{i}\right) m_{3 / 2}^{2} \\
A_{i j k} & =\left(3-\lambda_{i}-\lambda_{j}-\lambda_{k}\right) m_{3 / 2} \\
B_{0} & =\left(2-\lambda_{H_{u}}-\lambda_{H_{d}}+\rho\right) m_{3 / 2}
\end{aligned}
$$

and $\mu$ term is proportional to $1-\rho$. To make that the Higgsino mass $\mu$ is proportional to $m_{3 / 2}$, we need $\lambda_{H_{u}}+\lambda_{H_{d}}=2 \rho$. The gravitino mass is $m_{3 / 2}=\frac{|W|}{M_{P}^{2}} \frac{1}{(T+\bar{T})^{3 / 2}}$. The modular weights $\lambda_{i}$ and $\rho$ are determined in a concrete model [17, 18].

The gauge kinetic function to determine the gaugino mass is

$$
f_{A}=k_{A} T^{\ell_{A}}
$$

In our assumption, every weak scale mass parameter is proportional to one dimensionful mass. In order to achieve this, the gauge kinetic function should depend only on the real part of $T$. Then the modular weight $\ell_{A}$ needs to be 1 (or 0 ). Therefore, all (kinetic normalized) gaugino masses are unified at the boundary, while the gauge coupling constants can be different since $k_{A}$ can be different. The gaugino mass is same as the gravitino mass at the cutoff scale.

If there are fields which acquire heavy scale VEVs such as GUT Higgs fields, these fields need to be inside the log as in Eq.(20) so that the flat potential is not destabilized.

Even if the potential is flat at the tree-level, the quantum effects may destroy the flatness [17]. The dangerous term which destabilizes the electroweak scale is $\Lambda^{2} \operatorname{Str} M^{2} /\left(32 \pi^{2}\right)$, where $\Lambda$ is a cutoff scale. The supertrace is proportional to $m_{3 / 2}^{2}$ and thus, it destroys the dynamical determination of $m_{3 / 2}$ by electroweak potential. In a simplest case, $\operatorname{Str} M^{2}$ is negative, and then the gravitino mass goes to infinity. Therefore, $\operatorname{Str} M^{2}$ needs to be zero including moduli and the hidden sector fields. Here after, we assume that the supertrace is zero, which can be realized. 
Though we have to forbid the $\Lambda^{2} m_{3 / 2}^{2}$ term, there can be a harmless correction such as $\alpha m_{3 / 2}^{4}$ term in the potential. Such a term can arise due to Casimir effects which is related to the SUSY breakings, or due to a correction in the Kähler potential [17]

$$
-3 \ln (T+\bar{T}) \rightarrow-\ln \left((T+\bar{T})^{3}+c\right)
$$

When such a correction in the potential is taken into account, the result in the previous section is modified. The potential with the $\alpha m_{3 / 2}^{4}$ term is given, naively, as

$$
V \propto-Q_{V}^{4}\left(\ln \frac{Q_{0}}{Q_{V}}\right)^{2}+\bar{\alpha} Q_{V}^{4},
$$

where $\bar{\alpha}$ is proportional to $\alpha$. Then, minimizing the potential with respect to $Q_{V}$, we obtain

$$
\ln \frac{Q_{0}}{Q_{V}}=\frac{1+\sqrt{1+16 \bar{\alpha}}}{4},
$$

and $\ln \frac{Q_{0}}{Q_{V}}>\frac{1}{4}$ by using $\frac{\partial^{2} V}{\partial Q_{V}^{2}}>0$. Therefore, we write

$$
\left.M_{Z}^{2} \gtrsim \frac{1}{2}\left(1-\ln k^{2}\right) \frac{d m_{2}^{2}}{d \ln Q}\right|_{Q=Q_{0}},
$$

which provides an upper bound of the overall SUSY breaking scale $\left(m_{1 / 2}\right)$ for given $\hat{m}_{0}, \hat{A}_{0}$, and $\tan \beta$. The upper bound of the gaugino mass at the minimum is about $\sqrt{2}$ times compared to the $\alpha=0$ case Eq.(18). In figure 2, we show the numerical result of the minimization of $m_{1 / 2}$ with the experimental constraints. We emphasize that the no-scale bound we have obtained does not depend on the detail of the no-scale model constructed from string theory. We obtain the no-scale bound, as long as there is no a priori scale around the weak scale and the potential is flat.

In drawing the figure, we assume universal scalar mass $m_{0}$ and universal trilinear coupling $A_{0}$ at the GUT scale $\sim 2 \times 10^{16} \mathrm{GeV}$. We use 2-loop RGE between the GUT and the weak scale to determine the weak scale spectrum and 1-loop corrected potential for minimization. The 1-loop potential has a slight $Q$-dependence and it may change the result by a few percent. We choose the evaluation scale $Q$ to be about $500 \mathrm{GeV}$ so that the result is insensitive to $Q$. If the SUSY breaking terms are universal, $m_{0}$ and $A_{0}$ are related, $\hat{A}_{0}=3 \hat{m}_{0}^{2}$, due to Eqs.(22, 23), but we do not assume such relations in drawing the figure because of the reason which we will describe later.

As we have noted, the parameters are $\hat{m}_{0}, \hat{A}_{0}, \tan \beta$ and also a signature for $\mu$. We choose $\mu>0$ due to $b \rightarrow s \gamma$ constraint. We show the case for $\tan \beta=10$ at the minimal point of the potential so that the region is allowed by the $\operatorname{Br}[b \rightarrow s \gamma]$ which we take to be $2.2 \times 10^{-4}<\operatorname{Br}[b \rightarrow$ 


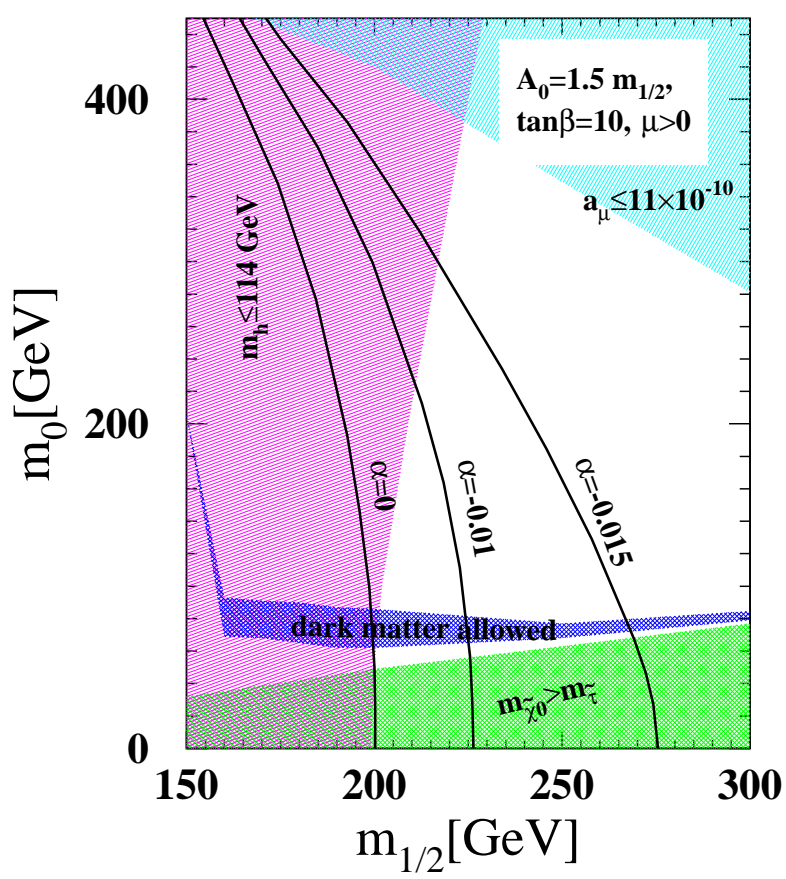

Figure 2: We show minimization contours of the potential for different values of $\alpha$ (defined in the text) in the mSUGRA parameter space. The blue narrow bands are allowed by dark matter constraints. The lightest Higgs mass $m_{H} \leq 114 \mathrm{GeV}$ is in the pink shaded region. $a_{\mu} \leq 11 \times 10^{-10}$ in the light blue shaded region.

$s \gamma]<4.5 \times 10^{-4}[19]$. We choose $\hat{A}_{0}=1.5$ to satisfy the bound on the lightest Higgs boson mass, as well as the $\operatorname{Br}[b \rightarrow s \gamma]$ constraint. Then, by changing $\hat{m}_{0}$, we obtain $m_{1 / 2}$ by numerical minimization of the potential, and the solid lines are drawn. The three lines corresponds to the value $\alpha=0,-0.01,-0.015$, where $\alpha$ is the coefficient of the correction of the potential $\alpha m_{3 / 2}^{4}$. When $\alpha>0$, the $m_{1 / 2}$ value at the minimal point of the potential becomes smaller. As one can understand from the discussion above, the minimal value of $m_{1 / 2}$ is almost determined by the ratio $m_{1 / 2} / Q_{V}$. The ratio is determined by the low energy spectrum, typically by stop mass. Therefore, the solid lines are naively obtained by the trajectories for constant average stop mass, and thus, they are elliptic curves in the $m_{0}-m_{1 / 2}$ plain. The solid lines are insensitive to the top quark mass, but depends on strong gauge coupling $\alpha_{3}$. We use $\alpha_{3}\left(M_{Z}\right)^{\overline{\mathrm{MS}}}=0.117$, and $m_{t}=172.7 \mathrm{GeV}$.

In the figure, we also draw the experimental constraints for the lightest Higgs boson mass [20], muon anomalous magnetic moment $a_{\mu}$ [21], dark matter [22]. We also show the region where neutralino is the lightest SUSY particle.

It is interesting to note that the no-scale allowed region is within the reach of the LHC, and in the mSUGRA model it is allowed by all the experimental constraints. It is also important to note that the dark matter $\tilde{\chi}_{1}^{0}-p$ cross-sections are (in $10^{-8} \mathrm{pb}$ ) 1.6-5, 1-2.7 and 0.3-1.8 for 
$\alpha=0,-0.01$ and -0.015 respectively. (The ranges in the cross-sections are obtained for the experimental range of $\sigma_{\pi N}$, strange quark content of proton and strange quark mass [23]). The recent upper limit on the neutralino proton cross-section is $5.5 \times 10^{-8} \mathrm{pb}$ from the XENON 10 experiment [24]. We see that the no-scale SUGRA allowed region will be probed very soon in these direct detection experiments.

The phenomenological constraints so far we have discussed are for mSUGRA models of soft SUSY breaking terms. Though we use the universal boundary conditions for $m_{0}$ and $A_{0}$ for simplicity to draw figure 2, the no-scale prediction does not depend on the detail of the boundary conditions Eqs.(22,23), as well as the cutoff scale very much because the prediction is determined by the low energy stop mass spectrum via Eqs.(18,29). On the other hand, the experimental constraints depends on the location of the cutoff scale as well as the universality conditions, especially for the dark matter allowed region and the stau LSP region.

The important prediction of the no-scale structure is Eq.(18) for true flat potential $\alpha=0$, and the bound is obtained from Eq.(29). Eq.(29) gives upper bound to stop mass, and therefore, generates upper bound to the gluino mass, as well. The gluino mass is bounded as $m_{\tilde{g}} \lesssim 730$ $\mathrm{GeV}$ when the lightest Higgs mass bound is taken into account. We show one example of the low energy spectrum:

\begin{tabular}{|c|c|c|c|c|c|c|}
\hline \hline parameters & \multicolumn{5}{|c|}{ masses in GeV } \\
\hline \hline$m_{0}, m_{1 / 2}, A_{0}, \tan \beta$ & $m_{\tilde{g}}$ & $m_{\tilde{t}_{1,2}}$ & $m_{h, H}$ & $m_{\tilde{\tau}_{1,2}}$ & $m_{\tilde{\chi}_{1,2,3,4}}$ & $m_{\tilde{\chi}_{1,2}^{ \pm}}$ \\
\hline $100,300,0,10$ & 730 & 480,670 & 114,450 & 151,243 & $125,233,410,430$ & 233,430 \\
\hline
\end{tabular}

Now we note the possible phenomenological solution in terms of the modular weights of the no-scale model in the following. (1) Choose the modular weight for slepton to be 1 in order not to overclose the universe 2 , and the cutoff scale is chosen to be around a few times $10^{17}$ GeV to avoid stau LSP. (2) Choose the modular weights for squarks and up-type Higgs field to generate the trilinear coupling with suitable values to satisfy the lightest Higgs boson mass bound and the $b \rightarrow s \gamma$ constraint especially for the case of true flat no-scale potential $\alpha=0$.

\section{Conclusion and Discussion}

Since the LEP and the Tevatron data do not show any direct evidence for SUSY, the SUSY particle mass scale has become larger compared to the $M_{Z}$ scale, therefore a little hierarchy is created between this scale and the $Z$ boson mass scale. In order to implement the little hierarchy, two apparently unrelated scales $Q_{0}$ (where the electroweak symmetry breaks radiatively) and $Q_{S}$ (the scale where the correct $Z$ boson mass gets produced) need to be close satisfying

\footnotetext{
${ }^{2}$ There may be an exception when the Boltzmann equation is modified [25].
} 
$Q_{S}<Q_{0}$, and the closeness is characterized by stop mass spectrum. In this paper, we have investigated the no-scale SUSY breaking models and found that the dynamical determination of the SUSY breaking scale in these models provides a natural solution of the little hierarchy. The two scales $Q_{0}$ and $Q_{S}$ get related in the no-scale model since the electroweak symmetry breaking vacuum also fixes $Q_{S}$ as a minimal of the electroweak potential. Since the potential, minimized by Higgs VEVs, is naively proportional to $-m_{Z}^{4}$, a larger overall scale is favored and a large $Q_{S}$ provides a small value for the potential. However, when $Q_{S}$ becomes very close to $Q_{0}$ (which is independent of the overall scale), the $Z$ boson mass becomes smaller by definition and the potential becomes larger. As a result, $Q_{S}$ is stabilized just below the scale $Q_{0}$.

We considered a no-scale potential where the potential is flat up to the gravitino mass and assumed that all the weak scale parameters are proportional to a single scale, which is natural in no-scale supergravity models. Then we found that the lighter stop and gluino masses can be as large as 480 and $730 \mathrm{GeV}$ respectively. These masses can be easily accessed at the LHC. Further, the parameter space is allowed by the Higgs mass bound and the $\operatorname{Br}[b \rightarrow s \gamma]$ using the mSUGRA boundary conditions. It is also interesting to note that the dark matter detection cross section is in the range from 0.3 to $5 \times 10^{-8} \mathrm{pb}$. The future dark matter detection experiments can easily probe these cross-sections. The model also can be fit with proper modular weight factors for the quark and lepton fields.

We now note what happens when we do not assume the single scale proportionality factor for the parameters. Suppose that both $\mu$ and $m_{1 / 2}$ are free and $\hat{m}_{0}$ and $\hat{A}_{0}$ are fixed. Then $Q_{0}$ can be changed by varying $\mu$, while the scale $Q_{H}$, where $M_{H}^{2}$ becomes zero, is independent of $\mu$ and $m_{1 / 2}$. As in the case where $Q_{0}$ is fixed in the single scale proportionality, $Q_{S}$ can be as large as $Q_{H}$ (but $Q_{S}<Q_{H}$ ). By definition, $Q_{S}<Q_{0}<Q_{H}$ is satisfied, and thus, all three scales are close at the minimal value of the potential. The closeness of $Q_{0}$ and $Q_{H}$ means that $\mu$ is small by definition and we find $\mu<M_{Z}$ at the minimal point, which is already excluded by the chargino mass bound. When both $\mu$ and $B$ are free from the other SUSY breaking parameters, one finds a non-stabilized direction to the $D$-flat direction. Thus the $\mu$ - $B$ ratio should be fixed in this case. Therefore, the single scale proportionality is a rational assumption in no-scale models.

In no-scale models, the potential for moduli $T$ is almost flat even if we include the electroweak potential, and therefore, the mass of the moduli is tiny, i.e., $m_{T} \sim m_{W}^{2} / M_{P} \sim 10^{-5}$ $\mathrm{eV}$. This moduli mass $m_{T} \sim m_{W}^{2} / M_{*}$ (where $M_{*}$ is a fundamental scale) does not depend on the detail of the model when the no-scale structure is broken by the radiative effect. Such light moduli overclose universe if the misaligned of the moduli from its minimal value is of the $O\left(M_{P}\right)$ after the inflation [26]. In order to avoid this problem, the misalignment from the 
minimal value should be much less than the Planck scale [27]. In other words, the moduli can be a part of dark matter. There are other interesting cosmological implications of no-scale model [28], which are out of scope of this paper.

Another point about the no-scale model is that the electroweak potential is $-O\left(m_{W}^{4}\right)$ at the minimum. Therefore, we need to add possible contribution to cancel the vacuum energy. Such contribution can come from other stabilized moduli or hidden sector fields from $F$ or $D$ term. However, such fields may destroy the no-scale structure. In general, such contribution generates

$m_{X}^{4-\gamma} m_{3 / 2}^{\gamma}$ term in the potential. For example, we obtain $\gamma=4 / 3$ when we use a hidden field with modular weight 1 . When $m_{X}$ is around the weak scale, it can avoid the destabilization of the no-scale electroweak potential and the vacuum energy can be canceled. However, by $m_{3 / 2}$ minimization, such positive terms require the overall scale to be smaller which is disfavored by experimental results. In order to make the model viable, we need to make $\gamma=0$, which is possible from a $D$-term contribution, so that the correction to tune vacuum energy should not depend at all on no-scale moduli $T$.

\section{Acknowledgments}

This work was supported in part by the DOE grant DE-FG02-95ER40917.

\section{References}

[1] K. Inoue, A. Kakuto, H. Komatsu and S. Takeshita, Prog. Theor. Phys. 68, 927 (1982); Prog. Theor. Phys. 71, 413 (1984); L. E. Ibanez and G. G. Ross, Phys. Lett. B 110, 215 (1982); L. E. Ibanez, Phys. Lett. B 118, 73 (1982); Nucl. Phys. B 218, 514 (1983); J. R. Ellis, D. V. Nanopoulos and K. Tamvakis, Phys. Lett. B 121, 123 (1983); J. R. Ellis, J. S. Hagelin, D. V. Nanopoulos and K. Tamvakis, Phys. Lett. B 125, 275 (1983); L. Alvarez-Gaume, J. Polchinski and M. B. Wise, Nucl. Phys. B 221, 495 (1983).

[2] H. Goldberg, Phys. Rev. Lett. 50, 1419 (1983); J. R. Ellis, J. S. Hagelin, D. V. Nanopoulos, K. A. Olive and M. Srednicki, Nucl. Phys. B 238, 453 (1984).

[3] K. Choi, K. S. Jeong and K. i. Okumura, JHEP 0509, 039 (2005) hep-ph/0504037; K. Choi, K. S. Jeong, T. Kobayashi and K. i. Okumura, Phys. Lett. B 633, 355 (2006) hep-ph/0508029]; hep-ph/0612258.

[4] R. Kitano and Y. Nomura, Phys. Lett. B 631, 58 (2005) hep-ph/0509039; Phys. Lett. B 632, 162 (2006) hep-ph/0509221]; Phys. Rev. D 73, 095004 (2006) hep-ph/0602096; 
hep-ph/0606134; Y. Nomura and D. Poland, Phys. Lett. B 648, 213 (2007) hep-ph/0611249.

[5] R. Dermisek and H. D. Kim, Phys. Rev. Lett. 96, 211803 (2006); R. Dermisek, H. D. Kim and I. W. Kim, JHEP 0610, 001 (2006).

[6] G. F. Giudice and R. Rattazzi, Nucl. Phys. B 757, 19 (2006) [hep-ph/0606105].

[7] B. Dutta and Y. Mimura, Phys. Lett. B 648, 357 (2007) hep-ph/0702002.

[8] E. Cremmer, S. Ferrara, C. Kounnas and D. V. Nanopoulos, Phys. Lett. B 133, 61 (1983); J. R. Ellis, A. B. Lahanas, D. V. Nanopoulos and K. Tamvakis, Phys. Lett. B 134, 429 (1984).

[9] J. R. Ellis, C. Kounnas and D. V. Nanopoulos, Nucl. Phys. B 241, 406 (1984); Nucl. Phys. B 247, 373 (1984); Phys. Lett. B 143, 410 (1984); A. B. Lahanas and D. V. Nanopoulos, Phys. Rept. 145, 1 (1987).

[10] Y. Chikira and Y. Mimura, hep-ph/0005231.

[11] J. R. Ellis, K. Enqvist, D. V. Nanopoulos and F. Zwirner, Mod. Phys. Lett. A 1, 57 (1986); R. Barbieri and G. F. Giudice, Nucl. Phys. B 306, 63 (1988); G. W. Anderson and D. J. Castano, Phys. Lett. B 347, 300 (1995) hep-ph/9409419]; K. L. Chan, U. Chattopadhyay and P. Nath, Phys. Rev. D 58, 096004 (1998) hep-ph/9710473.

[12] S. R. Coleman and E. Weinberg, Phys. Rev. D 7, 1888 (1973).

[13] I. Jack, D. R. T. Jones, S. P. Martin, M. T. Vaughn and Y. Yamada, Phys. Rev. D 50, 5481 (1994) hep-ph/9407291.

[14] J. L. Feng, K. T. Matchev and T. Moroi, Phys. Rev. Lett. 84, 2322 (2000) hep-ph/9908309; Phys. Rev. D 61, 075005 (2000) hep-ph/9909334.

[15] D. Z. Freedman, P. van Nieuwenhuizen and S. Ferrara, Phys. Rev. D 13, 3214 (1976); S. Deser and B. Zumino, Phys. Lett. B 62, 335 (1976); A. H. Chamseddine, R. Arnowitt and P. Nath, Phys. Rev. Lett. 49, 970 (1982).

[16] R. Barbieri, S. Ferrara and C. A. Savoy, Phys. Lett. B 119, 343 (1982); L. J. Hall, J. D. Lykken and S. Weinberg, Phys. Rev. D 27, 2359 (1983); P. Nath, R. Arnowitt and A. H. Chamseddine, Nucl. Phys. B 227, 121 (1983); For a review, see H. P. Nilles, Phys. Rept. 110, 1 (1984). 
[17] S. Ferrara, C. Kounnas and F. Zwirner, Nucl. Phys. B 429, 589 (1994) hep-th/9405188.

[18] P. G. Camara, L. E. Ibanez and A. M. Uranga, Nucl. Phys. B 689, 195 (2004) hep-th/0311241]; D. Lust, S. Reffert and S. Stieberger, Nucl. Phys. B 706, 3 (2005) hep-th/0406092; ; Nucl. Phys. B 727, 264 (2005) hep-th/0410074]; L. E. Ibanez, Phys. Rev. D 71, 055005 (2005) hep-ph/0408064; A. Font and L. E. Ibanez, JHEP 0503, 040 (2005) hep-th/0412150].

[19] M. S. Alam et al. [CLEO Collaboration], Phys. Rev. Lett. 74, 2885 (1995).

[20] R. Barate et al. [LEP Working Group for Higgs boson searches], Phys. Lett. B 565, 61 (2003); W. M. Yao et al. [Particle Data Group], J. Phys. G 33, 1 (2006).

[21] G. W. Bennett et al. [Muon $g-2$ Collaboration], Phys. Rev. Lett. 92, 161802 (2004) hep-ex/0401008; S. Eidelman, Talk at ICHEP 2006, Moscow, Russia.

[22] D. N. Spergel et al. [WMAP Collaboration], Astrophys. J. Suppl. 148, 175 (2003) astro-ph/0302209;; astro-ph/0603449.

[23] E. Accomando, R. Arnowitt, B. Dutta and Y. Santoso, Nucl. Phys. B 585, 124 (2000) hep-ph/0001019.

[24] Talk by E. Aprile at APS 07, Jacksonville, USA.

[25] A. B. Lahanas, N. E. Mavromatos and D. V. Nanopoulos, hep-ph/0608153; Phys. Lett. B 649, 83 (2007) hep-ph/0612152.

[26] J. R. Ellis, N. C. Tsamis and M. B. Voloshin, Phys. Lett. B 194, 291 (1987).

[27] K. Choi, E. J. Chun and H. B. Kim, Phys. Rev. D 58, 046003 (1998) hep-ph/9801280.

[28] M. Endo, K. Kadota, K. A. Olive, F. Takahashi and T. T. Yanagida, JCAP 0702, 018 (2007) [hep-ph/0612263]. 